\title{
Developmental prosopagnosia and the Benton Facial Recognition Test
}

\author{
Bradley C. Duchaine, PhD; and Ken Nakayama, PhD
}

\begin{abstract}
The Benton Facial Recognition Test is used for clinical and research purposes, but evidence suggests that it is possible to pass the test with impaired face discrimination abilities. The authors tested 11 patients with developmental prosopagnosia using this test, and a majority scored in the normal range. Consequently, scores in the normal range should be interpreted cautiously, and testing should always be supplemented by other face tests.
\end{abstract}

NEUROLOGY 2004;62:1219-1220

The Benton Facial Recognition Test (BFRT) is a commercially available test used by neurologists and neuropsychologists to assess face recognition abilities. ${ }^{1}$ Because the test is easy to administer and has extensive normative data, it is commonly used for clinical and research purposes. ${ }^{1}$ However, multiple lines of evidence suggest that individuals with impaired face recognition can score in the normal range on the test. To investigate this possibility, we have tested a relatively large sample of patients with developmental prosopagnosia using the BFRT.

The BFRT has a short form with 27 possible points and a long form with 54 possible points. On each item, subjects are presented with a target face above six test faces, and they are asked to indicate which of the six images match the target face. Male and female faces are used, and the faces are closely cropped so that no clothing and little hair are visible. The faces are centered within a black background, and the entire image is $\sim 6.5 \mathrm{~cm} \times 6.5 \mathrm{~cm}$. For the first six items, only one of the six test faces displays the target individual, and the target image and the test image are identical. In the next seven items, three of the test faces match the target face, and the poses for the test images are different from the target image. When the long form is administered, subjects are presented with nine more items. There are three matches per item and test faces are presented with different lighting conditions. There is a table for converting short form scores to long form scores, and scores of $\geq 41$ are classified as normal. No time limits are placed on individual items or the test as a whole.

Studies of patients with prosopagnosia and normal subjects suggest that normal scores on the BFRT do not require normal face recognition abilities. Two patients with prosopagnosia were reported who scored in the normal range on the BFRT, ,2,3 and these subjects reported that their performance relied heavily on feature matching rather than the holistic processing used by individuals with normal face recognition. ${ }^{4}$ Both subjects required more time than most subjects. ${ }^{2,3}$ In another study, normal subjects scored in the normal range despite the occlusion of most of the facial information. ${ }^{5}$ The subjects were presented a modified version of the BFRT in which only the eyebrows and hairlines were visible. Despite the limited information available, $59 \%$ of the subjects achieved normal scores on the short form, whereas $41 \%$ scored in the normal range on the long form.

We have tested individuals with developmental prosopagnosia using the BFRT to systematically assess whether patients with developmental prosopagnosia can pass it. Individuals with developmental prosopagnosia have had their impairment since early childhood. There are a variety of causes of developmental prosopagnosia, including genetic conditions,,$^{6,7}$ early brain damage, ${ }^{8}$ or in utero events. The condition sometimes causes prosopagnosics great social difficulty, but some are able to manage fairly well by using alternative routes for person recognition. ${ }^{9} \mathrm{We}$ have had the opportunity to test a relatively large sample of patients with developmental prosopagnosia during the past year because we have been contacted by $>150$ patients with self-reported prosopagnosia in the 18 months since we published a Web site discussing prosopagnosia (www.faceblind.org).

\footnotetext{
Methods. All of the subjects in our sample contacted us because they had serious face recognition problems in everyday life. To be included in the sample reported here, subjects had to score $>2$ SD below the mean on two of three tests of unfamiliar face discrimination. One of our subjects was one of the patients with prosopagnosia mentioned earlier who scored normally on the BFRT. ${ }^{2}$ We used two different unfamiliar face discrimination methods. Both required subjects to memorize a target face or a set of target faces and then discriminate the target from the nontarget faces. The three tests we used were the Face One in Ten Test and two versions of an old/new discrimination test (Old/New 1 and $\mathrm{Old} /$ New 2). These tests are discussed in detail elsewhere. ${ }^{7}$ Eleven patients with prosopagnosia were included in our sample, and six
} 


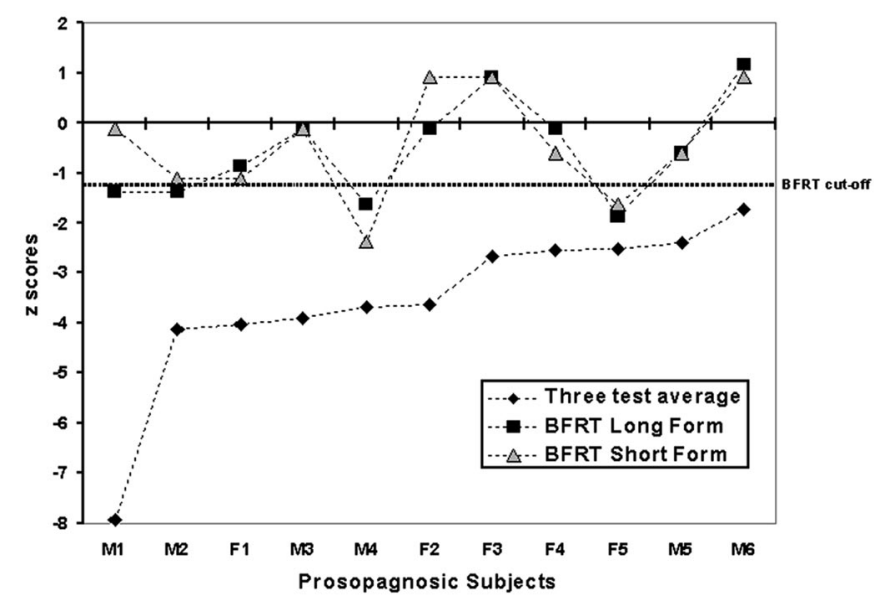

Figure. Performance of patients with developmental prosopagnosia on the face recognition tests as $Z$ scores: (control mean - individual score)/control SD. The three-test average is the mean of each subject's d' score on the Face One in Ten Test, Faces Old/New 1 Test and Faces Old/New 2 Test. Scores of $\geq 41$ are classified as normal by the Benton Facial Recognition Test, and we placed the dashed line labeled "Benton cut-off" at the corresponding $Z$ score.

were impaired on all three tests, whereas five were impaired on two tests. We converted their scores on the three tests to $\mathrm{Z}$ scores and averaged them to produce a $\mathrm{Z}$ score average for each individual (figure).

Results. We administered the full version of the BFRT so each patient with prosopagnosia produced a short form and a long form score. For the short form, the patients with prosopagnosia had a converted average score of 43.6 $(\mathrm{SD}=4.3)$. Scores of $\geq 41$ are considered normal, so this average is well within the normal range. Of the 11 subjects, 9 had scores in the normal range. On the long form, the patients with prosopagnosia averaged $43.3(\mathrm{SD}=4.0)$. Seven subjects had scores in the normal range. The figure shows the Z scores for the patients with prosopagnosia on both forms of the BFRT based on the normative data from a chapter by the test's creators. ${ }^{1}$ As mentioned previously, there are three different types of items. Subjects correctly matched all of the items with identical images. On the items with different poses, subjects attained $81.8 \%$ of the possible points; on items with different lighting, they attained $72.3 \%$ of the points.

Discussion. Our results make it clear that patients with developmental prosopagnosia are capable of performing normally on the BFRT. Like other patients with prosopagnosia who have scored normally, our subjects reported using the hairline, eyebrows, and other facial features. Most patients with prosopagnosia also required more time to complete the test than normal subjects we have tested. However, some patients with prosopagnosia responded quickly. Because patients with developmental prosopagnosia have had a lifetime to develop strategies, they may be especially adept at feature matching; therefore, patients with acquired prosopagnosia may not be as capable with this strategy. However, the results showing that normal subjects can successfully match the eyebrows and hairline of the BFRT faces indicate that extensive experience is not necessary to effectively use this strategy. ${ }^{5}$

Because normal scores on the BFRT do not demonstrate normal face discrimination, clinicians using the BFRT should interpret normal scores cautiously and should supplement their testing with other tests of face recognition. The development of time norms may allow the BFRT to be retained as an effective test, but some of our subjects were able to feature match rapidly. ${ }^{5}$ Any test with simultaneous presentation of the target face and the test faces will be vulnerable to a feature-matching strategy; therefore, it will probably be necessary to develop tests with a memory component to test face recognition. Note that our findings do not imperil the interpretation of impaired BFRT scores. A score below the normal cutoff indicates that a subject was not able to recognize the faces with a normal, holistic strategy or with a feature-matching strategy. ${ }^{4}$

Results from the BFRT have been widely used in neuropsychological studies of brain-damaged patients, and some of these case studies provided important support for theoretical models of face recognition. In particular, dissociations between familiar face recognition (e.g., celebrities and acquaintances) and unfamiliar face recognition in patients with acquired prosopagnosia led Bruce and Young ${ }^{10}$ to suggest that these two types of recognition are handled by separate processes in their model of face processing. However, most patients showing normal performance on unfamiliar face tasks with impaired performance on familiar face tasks were tested with the BFRT or other similar tests that allow for feature matching. ${ }^{5}$ Given our results and other evidence suggesting that normal scores do not necessarily indicate normal face recognition abilities, support for this dissociation should be re-evaluated.

\section{Acknowledgment}

The authors thank the subjects for their interest and participation.

\section{References}

1. Benton AL, Sivan AB, Hamsher K, Varney NR, Spreen O. Contributions to Neuropsychological Assessment. New York: Oxford University Press, 1994.

2. Duchaine BC. Developmental prosopagnosia with normal configural processing. Neuroreport 2000;11:79-83.

3. Nunn JA, Postma P, Pearson R. Developmental prosopagnosia: should it be taken at face value? Neurocase 2001;7:15-27.

4. Farah MJ, Wilson KD, Drain HM, Tanaka JR. What is "special" about face recognition? Psychol Rev 1998;105:482-498.

5. Duchaine BC, Weidenfeld A. An evaluation of two commonly used tests of unfamiliar face recognition. Neuropsychologia 2003;41:713-720.

6. deHaan EHF. A familial factor in the development of face recognition deficits. J Clin Exp Neuropsychol 1999;21:312-315.

7. Duchaine BC, Nieminen-von Wendt T, New J, Kulomaki T. Dissociations of visual recognition in a developmental agnosic: evidence for separate developmental processes. Neurocase 2003;9:380-389.

8. Barton JJS, Cherkasova M, Press DZ, Intriligator J, O'Connor M. Developmental prosopagnosia: a study of three patients. Brain Cogn 2003; $51: 12-30$.

9. Galaburda A, Duchaine B. Developmental disorders of vision. Neurol Clin 2003;21:603-615.

10. Bruce V, Young AW. Understanding face recognition. Br J Psychol 1986;77:305-327. 